Note: this is a draft of the journal article:

Worsley, P., Voegeli, D. (2013) Back to basics: biophysical methods in tissue viability research. Journal of Wound Care, 22(8): 434-439

The final, fully proofed and peer-reviewed journal article is available from the publisher online, via the following link:

http://www.internurse.com/cgibin/go.pl/library/article.cgi?uid=100187; article=JWC_22_8_434_439 


\title{
Back to basics: biophysical methods in tissue viability research
}

\author{
Peter Worsley PhD, BSc, MCSP - Senior Research Fellow \\ David Voegeli PhD, BSc, RN - Senior Lecturer
}

Address for Correspondence:

Dr David Voegeli

Continence Technology \& Skin Health Cluster,

Faculty of Health Sciences,

University of Southampton,

A Level (MP11) South Academic Block,

University Hospital Southampton,

Tremona Road,

Southampton. UK.

SO16 6YD.

Tel: $+44(0) 2380777222$ Extn: 3162.

Fax: +44 (0)2380796922.

Email: D.Voegeli@soton.ac.uk

Word Count: 3484

\section{Introduction}

The continued growth of research in the areas of tissue viability and wound care has led to the development of an equally diverse range of techniques to assess wounds and provide some quantitative measure of healing. These range from highly sophisticated imaging techniques, through to less sophisticated measurements of wound area. This variety often makes it difficult to fully understand the methods used, or to compare the results of different studies. Although a full outline of every single possible biophysical measurement 
technique is beyond the scope of this paper, there are several well established methods used in tissue viability research that have begun to cross over into clinical practice, particularly in the areas of burns, plastic surgery, and pressure ulcer prevention and management, and it is these that will be focused on.

\section{Laser Doppler flowmetry / imaging}

Laser Doppler flowmetry (LDF) developed in the 1970s as a non-invasive method to measure blood flow in the microvasculature (Stern, 1975) and was soon followed by the development of laser Doppler imaging (LDI). A more recent development in this area is laser speckle contrast imaging, enabling high resolution video imaging of blood flow over a wide area, which is able to detect rapid transient changes in blood flow not possible with the other systems. For a concise review of the developments in laser Doppler methods see Rajan et al., (2009).

\section{How does it work?}

Both techniques are based on measuring the frequency shift that occurs when a low-power laser beam hits moving red blood cells in the vasculature. The spectral purity of the laser enables these small frequency shifts to be detected, and quantified to produce an indirect measurement of blood flow. In LDF systems the laser beam is usually delivered by a fibre optic cable fixed to the tissue under examination, and the reflected /scattered beam fed back to a photo detector for analysis (Fig 1). Whilst LDI systems do not require any contact with the wound or tissue, and use a mirror system to move the laser beam over a predetermined area, and feedback the resulting backscattered light to the photo detector. The signal is then converted into a colour image representing blood flow over a larger area than can be achieved with LDF (Fig 2).

Interpretation of LDF and LDI.

The Doppler-shifted component of the laser beam, caused by moving red blood cells, and the non-shifted component from the tissue is detected by the photo detector and processed via inbuilt software to produce a measure known as flux (a product of the red blood cell concentration and their mean velocity) measured in arbitrary units (AU) or perfusion units (PU). Although this is an arbitrary value and not an absolute measure of blood flow, flux 
correlates with perfusion and is regarded as an indirect measure of overall blood flow in a tissue. In simple terms high flux values indicate greater blood flow and low values reduced flow.

In LDI systems the processing software enables the numerous data points to be presented as a colour 'map' of perfusion, which may be a single image or a series of images collected over a pre-set time-course.

\section{Disadvantages}

LDF systems have several main disadvantages that need to be taken into account. Skin blood perfusion is known to vary widely between sites, even over relatively small distances. Thus the fact that LDF involves a single point measurement can lead to wide variation, making it important to ensure measurements are taken at exactly the same location. Movement of the patient or of the fibre optic cables during measurement can lead to artefact, and produce erroneously high flux readings. Also the fact that the LDF probe has to be in direct contact with the tissue being monitored may make it unsuitable for use in open wounds. In contrast LDI avoids most of these disadvantages. As LDI scans over a larger area, collecting a greater number of data points, any variability is greatly reduced. Also the fact that measurement does not involve contact with the skin or wound makes it more acceptable for use in open wounds. The potential for movement artefact is greatly reduced by the absence of cables, although patient movement can disrupt the quality of the data obtained, and needs to be avoided.

\section{Clinical Application}

Although laser Doppler techniques have mainly been developed and used as research tools, they have established themselves in certain clinical settings. They are particularly useful in areas where a detailed knowledge of microcirculatory function is needed, such as wounds caused by burns, following plastic surgery or in wounds in conditions with a known underlying microvascular dysfunction (e.g. diabetes, venous leg ulcers). In burns patients the use of LDI has been shown to accurately determine the burn depth as well as being a reliable and sensitive tool for predicting healing and guiding surgical intervention (Pape et 
al., 2012; Gill 2013). In plastic surgery LDF and LDI has been extensively used to monitor perfusion in free skin flaps and has proved useful in providing early indications of reduced perfusion and flap vulnerability before clinically observed signs become apparent. However it is not just burns and plastics where these techniques have established themselves, but reports of use can be found across many other branches of surgery, from transplantation to intraoperative use in bowel surgery and orthopaedics.

\section{Transcutaneous gas monitoring}

Commercial transcutaneous monitors first appeared in the late 1970's and resulted from an increase in physiological understanding and technical innovation. The first 'combined' transcutaneous pO2 and pCO2 monitoring system was described in 1979 (Parker et al., 1979), and was the forerunner of today's transcutaneous monitors.

\section{How does it work?}

$\mathrm{TcPO}_{2}$ and $\mathrm{TCPCO}_{2}$ are monitored by a small sensor, which houses a $\mathrm{pH}$ electrode, a reference electrode, an electrolyte solution, a Teflon membrane, and a heating element. The calibrated sensor is attached to the skin by special sticky fixation rings. Some contact gel is placed in the well of the fixation ring before the sensor is secured to exclude air bubbles and provide good thermal contact with the skin. The heating element warms the skin to 42 $45^{\circ} \mathrm{C}$, which causes vasodilatation of the capillary loops, producing a massive increase in local skin blood flow. Increasing the temperature of the in-rushing blood causes a rightward shift of the oxyhaemoglobin dissociation curve, effectively increasing its pO2. At about $43.5^{\circ} \mathrm{C}$, there is a critical balance of these (and other) temperature effects. Provided this critical balance is not upset, the transcutaneous pO2 (tcpO2) at the skin surface agrees closely with the arterial pO2 (Bromley, 2008). The heat also dissolves the lipid structure of the dead keratinized cells which increases the permeability of the skin (Figure 3). Once the sensor has been applied, it takes about 10- 15 minutes for 'hyperaemisation' to take place: only then are the transcutaneous readings meaningful. 
When interpreting transcutaneous data it is important to understand normal values for TCPO2 and TcCO2. In the healthy population an average of $70 \mathrm{mmHg}$ (range $45-90 \mathrm{mmHg}$ ) has been described for TcPO2 (Dowd et al. , 1983) and an average of 38mmHg (range 30$45 \mathrm{mmHg}$ ) for TcPCO2 (Lagerkvist et al. , 2003). When sufficient pressure is applied over the skin and soft tissues the blood supply can be occluded causing local ischemia. This is reflected by a decrease in the $\mathrm{TcO} 2$ reading and in some cases an increase in $\mathrm{TcCO} 2$ (Chai and Bader 2013). Tissue viability thresholds for these changes have been described for pressure ulcer risk TcPO2 (Bogie et al. , 1995, Chai and Bader 2013) and wound healing (Arsenault et al. , 2012). However, these thresholds are advised not to be used in isolation, and should be viewed as one of many clinical parameters in a holistic evaluation in wound healing.

\section{Disadvantages of transcutaneous monitoring}

Transcutaneous monitoring is undoubtedly more complicated to use than other techniques. The sensor has to be re-sited regularly (at least four-hourly) to minimise risk of skin damage. Erythema can occur, but quickly fades once monitoring has stopped. There is a common misconception that transcutaneous monitoring inevitably leads to skin burns. Varying degrees of burns can result from the sensor's elevated temperature. Thin-skinned infants and patients with peripheral vascular impairment are especially at risk. Transcutaneous monitoring is also relatively expensive with consumables (calibration gas cylinders, membrane kits, and fixation kits) associated costs.

\section{Clinical Application in Wound Healing}

Transcutaneous gas monitoring is finding increasing application as a diagnostic tool to assess the peri-wound oxygen tension of wounds, ulcers, and skin flaps (Fife et al., 2009). $\mathrm{TcPO}_{2}$ measurements have been used to monitor wound healing in lower limb amputees (Arsenault, Al-Otaibi, 2012), foot ulcers (Niinikoski, 2003), burn scar healing (Brusselaers et al. , 2010), and non-healing ischemic extremity wounds (Grolman et al., 2001). A recent systematic review and meta-analysis by Arsenault et al (2012) highlighted that $\mathrm{TcPO}_{2}$ 
predicts healing complications of lower limb amputations, but its independent predictive value has not been determined. $\mathrm{TCPCO}_{2}$ has also been shown to be a useful indicator of wound healing. One study showed that in pressure ulcers it was found that carbon dioxide levels were negatively correlated to healing rates. However $\mathrm{TcPO}_{2}$ of peri ulcer was not statistically significantly related to healing rates (Black, 2000). When comparing transcuataneous measures to other modalities such as laser Doppler flowmetry it was shown to be more effective when evaluating wound healing after excision of extremity softtissue sarcomas (Conlon et al. , 1994).

\section{Interface Pressure Mapping.}

One of the key external risk factors for pressure ulcers is the exposure of prolonged periods of pressure at the interface between the support surface and the individual. In order to assess supportive devices for their efficacy in reducing both the magnitude and in some cases time (e.g. alternating air pressure mattress) various interface pressure mapping devices have been developed. These pressure-measuring systems have become an increasingly widely used assessment tool in wheelchair and seating clinics (Crawford et al., 2005, Sprigle, 2003) as well as research into mattresses and other support surfaces. The move from research applications to clinical utility has largely been due to manufacture-led improvements in both hardware and software (Bader et al., 2005). In addition, more intuitive software programs both increase the usability for the non-expert operator and allow real-time feedback on changing conditions at the seating support interface. There are several different types of pressure mapping devices on the market including electropneumatic, pneumatic, piezo-resistive and capacitive.

\section{Interpretation of interface pressure mapping}

Depending on the pressure mapping device being used there will be various outputs to assess. Factors include units of measurement (typically $\mathrm{mmHg}$ ), size and orientation of the pressure mapping device, resolution of the pressure mapping device, visual display format (e.g. contour plot, 3D plot [Figure 4]) and the posture/anatomy of the individual being measured. Research to date has shown many different ways of interpreting pressure mapping data. One of the key issues surrounding this analysis is the reduction of large data 
sets. Typically one of the latest pressure mapping systems could have $32 \times 32$ sensors giving 1024 readings. In addition, most mapping systems can record over a period of time at varying frequencies multiplying the amount of data collected from a given assessment. Commonly you see individuals using discrete values from these large data sets including;

- Peak value

- Average value

- Symmetry

- Highest 10 values

- Peak gradients (change in pressure over area)

A number of researchers have also used interface pressure thresholds of pressure in their analysis (Dover et al., 1992, Rithalia et al., 2000). However, there is still considerable debate and little consensus over what constitutes a safe interface pressure (Bader and Bouten, 2005). An often quoted cut-off figure for safe interface pressure is $32 \mathrm{mmHg}$, which is the capillary pressure as measured by Landis (Landis, 1930). This figure, however, was measured in 1930 and relates to the pressure required to occlude a nail fold capillary at heart level. It is difficult to see the relation of this pressure to the pressures needed to stop blood flow in the gluteal region, where there are complicated interactions between skin, fat, muscle and bone.

\section{Disadvantages of interface pressure mapping}

With any clinical assessment, an ideal technique would be reliable to use and valid/sensitive to the clinical outcome. Previous research has shown that pressure mapping can be reliable between clinicians (Stinson et al., 2003). However, this study assessed the visual ranking of the pressure map, not the actual numerical outputs. There remains a little or no robust evidence regarding the intra or inter-rater reliability of numerical outputs from pressure mapping systems. Subject variability, such as body weight, muscle tone, body fat content and skeletal frame size also influences interface pressure (Shelton et al., 1998). The subject themselves influence interface pressure in terms of how they get onto the support surface as well as how they position themselves on that support surface (Shelton 1998; Koo et al., 
1996, Swain and Bader, 2002). In addition, pressure mapping systems are highly dependent on material properties of the pressure transducer, soft tissue and the support surface which may cause variability in data output. Factors such as the size of sensor mat, the number of sensors, and the sensitivity of the system will influence the resolution, accuracy, and reliability of the measured pressure values (Eitzen, 2004).

Research has also shown that the translation of external pressure to the internal soft tissues is not a linear relationship and it has been shown that in muscle tissue just under bony prominences large tissue strains can be observed, because of stress concentrations around the bone-muscle interface (Linder-Ganz et al., 2007). Tissue viability under compression depends not only on the magnitude of mechanical loads but also on the time of exposure to loads (Gefen, 2007). With this in mind, pressure measurements alone is not sufficient to alert the clinician to potential areas of tissue breakdown (Reenalda et al., 2009).

\section{Clinical Application in Wound Healing}

There are two primary applications advocated for clinical use; firstly in patient education, and secondly to provide feedback for cushion selection for a given individual (Bader and Bouten, 2005). In terms of patient education, pressure mapping systems can give real time biofeedback as to how pressure is distributed over the area of interest. For example, if a patient is leaning to one side the pressure mapping assessment can show this in changing peaks of pressure and can help the patient address the postural asymmetry. Interface pressure can be used to identify areas of unacceptably high pressures and to ensure a site is adequately off-loaded during posture changes or a weight shift (Sprigle and Sonenblum, 2011). A good example of its clinical efficacy was shown by Dover et al (1992) in a large spinal injury unit. Here the pressure mapping tool was used to show patients how changing posture varies the distribution of pressure and helped to inform staff of the optimal internal pressures of air cushions (Dover and Pickard, 1992). The implementation of such a clinic has proved successful and resulted in a reduction of over $50 \%$ both in the incidence of pressure ulcers and in the admission rate due to ulcers, when compared with studies from other spinal units.

\section{Transepidermal Water Loss (TEWL)}


Transepidermal water loss (TEWL) is regarded as one of the most important parameters for characterizing skin barrier function (Kottner et al. , 2013). TEWL is a flux density, ie a quantity of water per unit area of skin per unit time. The first examples of TEWL measures date back to the 1940s (Baker and Kligman, 1967), with subsequent development of techniques now resulting in several commercially available tools which are used internationally in dermatology practice and research. TEWL values are affected by the state and function of the stratum corneum (SC). There is now established evidence linking increased TEWL with skin barrier dysfunction (Imhof et al. , 2009).

\section{How does it work?}

Evaporation of water from the skin takes place as part of the normal skin metabolism. TEWL probes measure the density gradient of the water evaporation from the skin indirectly using sensors (temperature and relative humidity). TEWL is a measure of the flux density of condensed water diffusing from the deeper highly hydrated layers of the dermis and epidermis to the skin surface and is usually expressed in $\mathrm{g} / \mathrm{h} / \mathrm{m}^{2}$ (grams of water per square meter of skin per hour).

Currently there are no methods for measuring TEWL directly. Current techniques infer TEWL values from water vapor flux evaporating from the skin. TEWL can be measured according to different approaches (Farahmand et al. , 2009):

(i) Closed-chamber method, which measures the increase of relative humidity (RH) in a closed air chamber

(ii) Ventilated chamber method, which measures water picked up by a gas passing through the chamber

(iii) Open-chamber method, which utilizes an open capsule, and estimates the vapour pressure gradient from the difference in vapour pressure at the two fixed heights of measurement.

\section{Interpretation of TEWL}

TEWL outputs can be effected environmental factors such as humidity, temperature and the moisture content of the skin. Therefore, care must be taken when interpreting the resultant 
data. Two key conditions need to be established in order to record accurate and reliable TEWL measures;

1. TEWL must be the only source of water vapour (factors such as perspiration and surface water can effect measures)

2. Ensure complete evaporation from the skin surface. High TEWL measures correspond with high skin surface humidity, if this reaches saturation (100\% relative humidity) some transepidermal water would remain as moisture on the skin surface giving a false reading (too low).

When interpreting results from TEWL assessment it is also important to acknowledge measures will vary considerably between skin areas in differing anatomical locations due to the functional anatomy of the SC (Menon and Kligman, 2009, Mohammed et al. , 2012). In a recent systematic literature review and meta-analysis it was highlighted that not only was anatomical site related to changes in TEWL but also age, with individuals aged 65 years and above showing consistently lower TWEL than the younger population (Kottner, Lichterfeld, 2013).

\section{Disadvantages of TEWL}

The common limitation with all TEWL techniques is that they interfere with the microclimate overlying the surface of the skin. The main problem with open-chamber systems is their vulnerability to disturbance from ambient air movements. The major concern with the closed-chamber method is its inability to perform continuous measurements, as the accumulated water vapour needs to be purged after every reading. Comparative studies have been performed using the different TEWL measurement techniques, showing a significant correlation between each approach (Farahmand, Tien, 2009). However, the sensitivity of each system was shown to differ under varying skin conditions. In addition any TEWL device needs stringent and regular calibration to ensure accurate results (Pinnagoda et al. , 1990). As highlighted above TEWL is also very dependent on the repeatability of measuring the same anatomical site, and testing in the same environmental conditions. 
As highlighted previously TEWL values are affected by the state and function of the stratum corneum (SC). An aspect of wound healing can therefore be assessed by measuring the rate of recovery of the skin barrier function using TEWL measurements. It is therefore not surprised that research studies have used the technique extensively when assessing wound healing treatments (Nakamizo et al. , 2013) and is being advocated as part of assessment tools in wound and scar management (Brusselaers, Pirayesh, 2010, Schultz et al., 2005).

\section{Combining measures}

In order to assess both external biomechanical and internal physiological markers researcher have combined techniques, particularly in the area of pressure ulcer research. A recent study was conducted by Kim et al (2012), where interface pressures and transcutaneous blood gas measurements were taken during sitting and lying. Here they took measurements every 5 minutes for a total of 20 minutes. They found no difference between time point measurements and no significant correlations between interface pressure and $\mathrm{TcPO}_{2}$. However, they did find some asymmetries in measures taken from the left and right side of the ischium highlighting that multiple sites of evaluation should be taken (Kim et al., 2012). There are however, some questions as to whether 20 minutes is sufficient to evaluate physiological response to pressure given the known association of time. Often in ward environments patients are left in static positions for several hours.

\section{Summary}

Numerous biophysical measurement techniques exist that may be applied to tissue viability research. Most require use in carefully controlled environments and extensive training in their use and interpretation, making them unsuitable for direct application to clinical practice, except in specialist centres. However a few have made the transition from laboratory to bedside and have established themselves as useful tools in assessing, monitoring and guiding clinical therapy, particularly in complex wounds or those where there is a strong underlying degree of microvascular dysfunction. In these cases having a 
working knowledge of the basic principles of operation helps in interpreting clinical data and having a more critical eye when reading research reports. 


\section{References}

Arsenault KA, Al-Otaibi A, Devereaux PJ, Thorlund K, Tittley JG, Whitlock RP. The Use of Transcutaneous Oximetry to Predict Healing Complications of Lower Limb Amputations: A Systematic Review and Meta-analysis. Eur J Vasc Endovasc Surg. 2012;43:329-36.

Bader DL, Bouten C, Colin D, Oomens C. Pressure Ulcer Research: Current and Future Perspectives. 1 ed. New York: Springer; 2005.

Baker $\mathrm{H}$, Kligman AM. Measurement of transepidermal water loss by electrical hygrometry: Instrumentation and responses to physical and chemical insults. Arch Dermatol. 1967;96:441-52.

Black J. Tissue Oxygen Perfusion and Pressure Ulcer Healing. Plast Surg Nurs. 2000;20:10-4. Bogie KM, Nuseibeh I, Bader DL. Early progressive changes in tissue viability in the seated spinal cord injured subject. Paraplegia. 1995;33:141-7.

Bromley I. Transcutaneous monitoring - understanding the principles. Infant. 2008;4:95-8. Brusselaers N, Pirayesh A, Hoeksema H, Verbelen J, Blot S, Monstrey S. Burn scar assessment: A systematic review of objective scar assessment tools. Burns. 2010;36:1157-64.

Chai CY, Bader DL. The physiological response of skin tissues to alternating support pressures in able-bodied subjects. Journal of the Mechanical Behavior of Biomedical Materials.2013

Conlon KC, Sclafani L, Diresta GR, Brennan MF. Comparison of transcutaneous oximetry and laser Doppler flowmetry as noninvasive predictors of wound healing after excision of extremity solft-tissue sarcomas. Surgery. 1994;115:335-40.

Crawford SA, Strain B, Gregg B, Walsh DM, Porter-Armstrong AP. An investigation of the impact of the Force Sensing Array pressure mapping system on the clinical judgement of occupational therapists. Clin Rehabil. 2005;19:224-31.

Dover H, Pickard W, Swain I, Grundy D. The effectiveness of a pressure clinic in preventing pressure sores. Paraplegia. 1992;30:267-72.

Dowd GSE, Linge K, Bentley G. Measurement of Transcutaneous Oxygen Pressure in Normal and Ischaemic Skin. Journal of Bone and Joint Surgery [Br]. 1983;65:79-83.

Eitzen I. Pressure mapping in seating: a frequency analysis approach. Arch Phys Med Rehabil. 2004;85:1136-40.

Farahmand S, Tien L, Hui X, Maibach HI. Measuring transepidermal water loss: a comparative in vivo study of condenser-chamber, unventilated-chamber and open-chamber systems. Skin Research and Technology. 2009;15:392-8. 
Fife CE, Smart DR, Sheffield PJ, Hopf HW, Hawkins G, Clarke D. Transcutaneous oximetry in clinical practice: consensus statements from an expert panel based on evidence. Undersea Hyperb Med. 2009;36:43-53.

Gefen A. The biomechanics of sitting-acquired pressure ulcers in patients with spinal cord injury or lesions. International Wound Journal. 2007;4:222-31.

Gill P. The critical evaluation of laser Doppler imaging in determining burn depth. Int J Burn Trauma 2013;3(2):72-77.

Grolman RE, Wilkerson DK, Taylor JL, Allinson P, Zatina MA. Transcutaneous oxygen measurements predict a beneficial response to hyperbaric oxygen therapy in patients with nonhealing wounds and critical limb ischemia. Am J Surg. 2001;67:1072-9.

Imhof RE, De Jesus MEP, Xiao P, Ciortea LI, Berg EP. Closed-chamber transepidermal water loss measurement: microclimate, calibration and performance. International Journal of Cosmetic Science. 2009;31:97-118.

Kim JH, Wang XL, Ho CH, Bogie KM. Physiological measurements of tissue health; implications for clinical practice. International Wound Journal. 2012;Jan 30.

Koo TKK, Mak AFT, Lee YL. Posture effect on seating interface biomechanics: Comparison between two seating cushions. Arch Phys Med Rehabil. 1996;77:40-7.

Kottner J, Lichterfeld A, Blume-Peytavi U. Transepidermal water loss in young and aged healthy humans: a systematic review and meta-analysis. Arch Dermatol Res. 2013;305:31523.

Lagerkvist A-L, Sten G, Redfors S, Holmgren D. Repeated blood gas monitoring in healthy children and adolescents by the transcutaneous route. Pediatr Pulmonol. 2003;35:274-9. Landis EM. Micro-injection studies of capillary blood pressure in human skin. Heart. 1930;15:209-28.

Linder-Ganz E, Shabshin N, Itzchak Y, Gefen A. Assessment of mechanical conditions in subdermal tissues during sitting: A combined experimental-MRI and finite element approach. J Biomech. 2007;40:1443-54.

Menon GK, Kligman AM. Barrier Functions of Human Skin: A Holistic View. Skin Pharmacology and Physiology. 2009;22:178-89.

Mohammed D, Matts P, Hadgraft J, Lane M. Variation of Stratum Corneum Biophysical and Molecular Properties with Anatomic Site. AAPS J. 2012;14:806-12.

Nakamizo S, Egawa G, Doi H, Natsuaki Y, Miyachi Y, Kabashima K. Topical Treatment with Basic Fibroblast Growth Factor Promotes Wound Healing and Barrier Recovery Induced by Skin Abrasion. Skin Pharmacology and Physiology. 2013;26:22-9. 
Niinikoski J. Hyperbaric oxygen therapy of diabetic foot ulcers, transcutaneous oxymetry in clinical decision making. Wound Repair Regen. 2003;11:458-61.

Pape, S.A. Baker, R. D. Wilson, D. Hoeksema, H. Jeng, J.C. Spence, R.J. Monstrey, S Burn wound healing time assessed by laser Doppler imaging (LDI). Part 1: Derivation of a dedicated colour code for image interpretation Burns. Burns. 2012;38, 187-94.

Parker D, Delpy D, Reynolds EOR. Single electrochemical sensor for transcutaneous measurement of pO2 and pCO2. Birth Defects: Original Article Series. 1979;15:109-16.

Pinnagoda J, Tupkek RA, Agner T, Serup J. Guidelines for transepidermal water loss (TEWL) measurement. Contact Dermatitis. 1990;22:164-78.

Reenalda J, Jannink M, Nederhand M, Ijzerman M. Clinical Use of Interface Pressure to Predict Pressure Ulcer Development: A Systematic Review. Assist Technol. 2009;21:76-85.

Rithalia SV, Heath GH, Gonsalkorale M. Assessment of alternating-pressure air mattresses using a time-based pressure threshold technique and continuous measurements of transcutaneous gases. Journal of Tissue Viability. 2000;10:13-20.

Schultz G, Mozingo D, Romanelli M, Claxton K. Wound healing and TIME; new concepts and scientific applications. Wound Repair Regen. 2005;13:S1-S11.

Shelton F, Barnett R, Meyer E. Full-body interface pressure testing as a method for performance evaluation of clinical support surfaces. Appl Ergon. 1998;29:491-7.

Sprigle S. Interface pressure measurement: Applying research findings to clinical use. . Rehabilitation Engineering and Assistive Technology Society of North America (RESNA): 26th International Conference on Technology and Diversity. Atlanta, GA2003.

Sprigle S, Sonenblum S. Assessing evidence supporting redistribution of pressure for pressure ulcer prevention: A review. J Rehabil Res Dev. 2011;48:203-14.

Stinson MD, Porter-Armstrong AP, Eakin PA. Pressure mapping systems: reliability of pressure map interpretation. Clin Rehabil. 2003;17:504-11.

Swain I, Bader DL. The measurement of interface pressure and its role in soft tissue breakdown. Journal of Tissue Viability. 2002;12:140-6.

Rajan V, Varghese B, van Leeuwen T, Steenbergen W. Review of methodological developments in laser Doppler flowmetry. Lasers Med Sci.2009; 24:269-283. 


\section{Figures:}

Figure 1: Diagram outlining the basic working of an LDF system (Courtesy Moor Instruments Ltd)

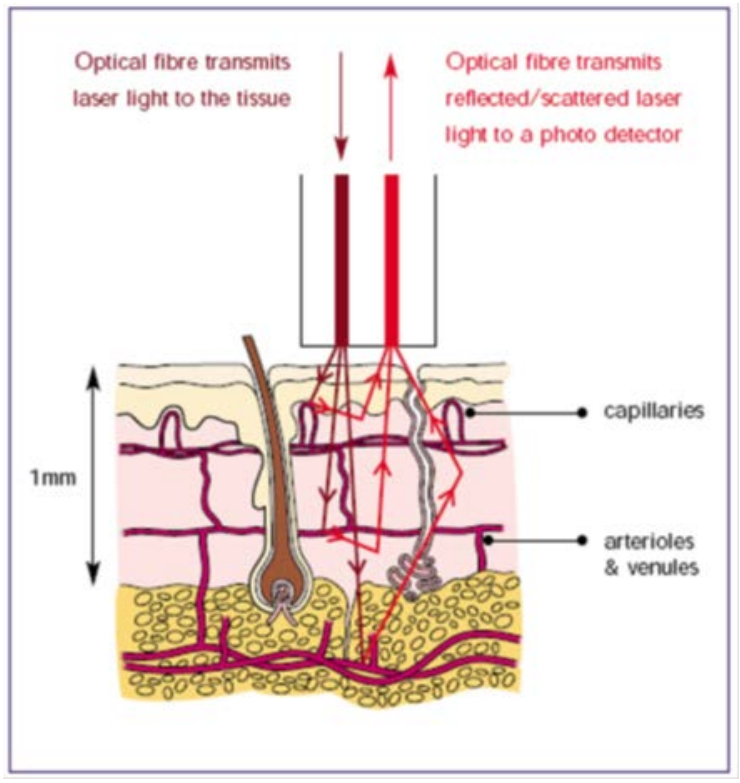

Figure 2: Diagram outlining the basic working of an LDI system (Courtesy Moor Instruments Ltd)

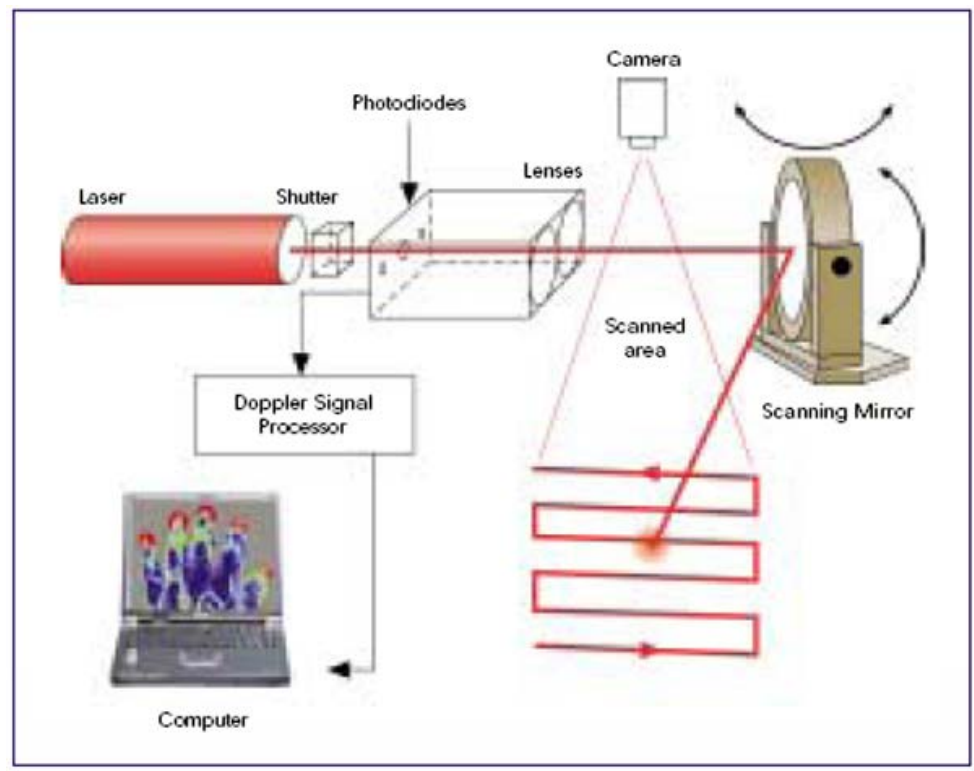


Figure 3: Basic mechanism of action of transcutaneous oxygen monitors

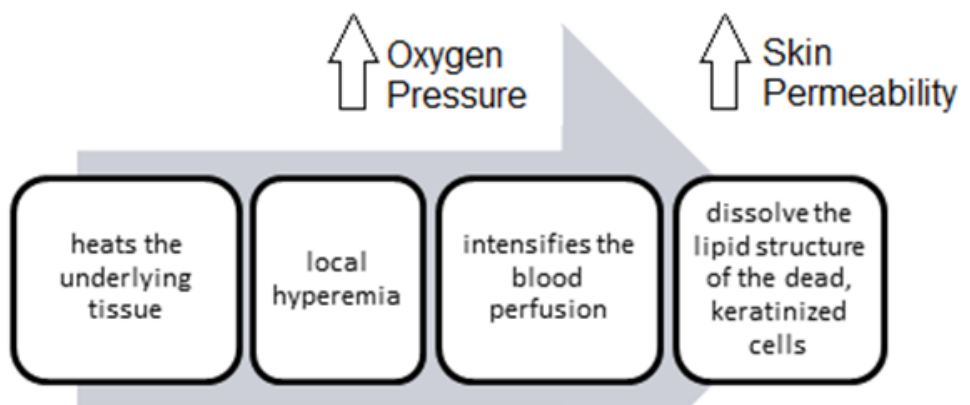

Figure 4: Example of an image generated from an interface pressure mapping system.

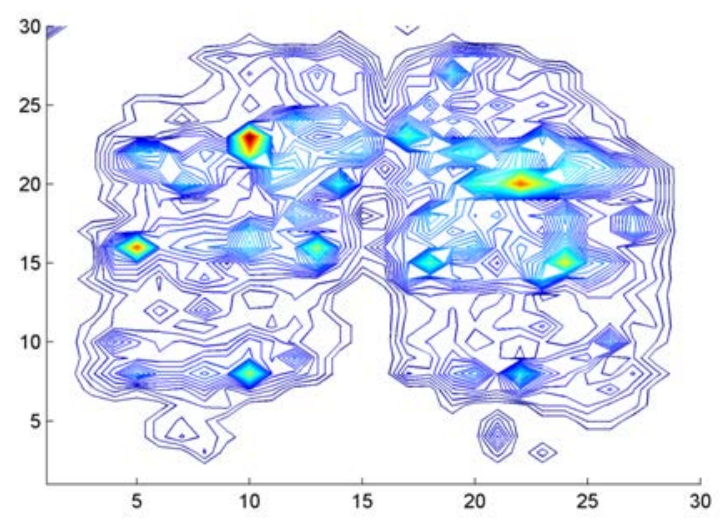

